\title{
CT Texture analysis and CT scores for characterization of fluid collections
}

\author{
Hans-Jonas Meyer ${ }^{1 *+}$, Benedikt Schnarkowski ${ }^{1+}$, Jakob Leonhardi ${ }^{1}$, Matthias Mehdorn ${ }^{2}$, Sebastian Ebel ${ }^{1}$, \\ Holger Goessmann ${ }^{1}$ and Timm Denecke ${ }^{1}$
}

\begin{abstract}
Background: Texture analysis derived from Computed tomography (CT) might be able to better characterize fluid collections undergoing CT-guided percutaneous drainage treatment. The present study tested, whether texture analysis can reflect microbiology results in fluid collections suspicious for septic focus.

Methods: Overall, 320 patients with 402 fluid collections were included into this retrospective study. All fluid collections underwent CT-guided drainage treatment and were microbiologically evaluated. Clinically, serologically parameters and conventional imaging findings as well as textures features were included into the analysis. A new CT score was calculated based upon imaging features alone. Established CT scores were used as a reference standard.

Results: The present score achieved a sensitivity of 0.78 , a specificity of 0.69 , area under curve (AUC 0.82). The present score and the score by Gnannt et al. (AUC 0.81) were both statistically better than the score by Radosa et al. (AUC 0.75). Several texture features were statistically significant between infected fluid collections and sterile fluid collections, but these features were not significantly better compared with conventional imaging findings.

Conclusions: Texture analysis is not superior to conventional imaging findings for characterizing fluid collections. A novel score was calculated based upon imaging parameters alone with similar diagnostic accuracy compared to established scores using imaging and clinical features.
\end{abstract}

Keywords: Texture analysis, CT, Fluid collection, Drainage treatment

\section{Background}

Fluid collections are common and can represent ascites, seroma, hematoma, biloma, lymphocele, or abscess depending on the localization and underlying cause [1-4]. The mortality rates associated with infected fluid collections range from $4 \%$ in treated cases to more than $80 \%$ in untreated cases [4]. Hence, early and correct diagnosis and treatment are crucial to determine the clinical outcome $[4,5]$. Many of these patients are

\footnotetext{
*Correspondence: Hans-jonas.meyer@medizin.uni-leipzig.de

${ }^{\dagger}$ Hans-Jonas Meyer and Benedikt Schnarkowski have contributed equally to this work.

${ }^{1}$ Department of Diagnostic and Interventional Radiology, University of Leipzig, Liebigstraße 20,04103 Leipzig, Germany

Full list of author information is available at the end of the article
}

suitable for Computed tomography (CT)-guided percutaneous drainage due to advances in CT-guided treatment, whereas surgery is only performed in few selected cases [5].

The important aspects to diagnose an infected fluid collection is clinical investigation, serological parameters, of most importance the inflammation parameter C-reactive protein (CRP) and lastly imaging modalities. CT is commonly performed in these patients to detect possible septic foci and to plan the following treatment.

Some imaging features were reported indicative of an infection of a fluid collection, including air entrapment, rim contrast media enhancement and Hounsfield unit $(\mathrm{HU})$ attenuation. However, these imaging features by 
itself cannot reliably exclude infection due to lack of a sufficient accuracy for correct diagnosis [3, 4].

Those imaging and clinical features lead to a proposed score by Gnannt et al., which utilized CT features, clinical and serological parameters to predict the likelihood of infected abdominal fluid collections undergoing CTguided drainage treatment in postsurgical patients [6]. In this study, the score achieved an excellent area under the curve of 0.96 in a validation cohort comprising 30 patients [6].

To this date, only one other study performed a validation analysis for this score and proposed a more accessible combination of imaging findings with only one clinical parameter, namely CRP to correctly diagnose infected fluid collections [4].

This proposed simplified score achieved an even higher accuracy than the first one by Gnannt et al. with a reported sensitivity of $93 \%$ and a specificity of $80 \%$ [4].

Texture analysis is a novel imaging analysis, which quantifies radiological images and can thereby provide imaging biomarkers [7-9]. Most commonly are images derived from Computed tomography (CT) due to its robust imaging acquisition and high availability in clinical routine [7-9]. In recent years, texture analysis was predominantly investigated in oncological imaging to better characterize tumor behavior [9]. Thus, it is acknowledged that texture analysis can give deeper insight into the microstructure of tissues. Presumably, texture analysis might also help to better characterize different components of fluid collections to discriminate hematoma and bacterial debris. However, texture analysis has not been employed for this purpose yet.

The principal hypothesis for this present work is that texture analysis might be able to reflect distinctive differences of fluid collections and might be a reliable tool to better characterize fluid collections and the need of drainage treatment. As a second part, the study performed a validation analysis for the two proposed scores to test the external validity and to validate, whether these scores can also be used in non-surgical patients and fluid collections of the pleura.

\section{Methods}

The radiological database of a university hospital was retrospectively screened for patients treated with CTguided percutaneous drainage between January 2017 and December 2020.

Inclusion criteria were sufficient contrast enhanced CT for septic evaluation within $24 \mathrm{~h}$ before the intervention, sufficient native planning $\mathrm{CT}$, available serological and microbiological evaluation. Overall, 738 patients were identified in the database, who were treated with a CTguided percutaneous drainage. Several patients had to be excluded for the following reasons: $\mathrm{n}=252$ patients with no contrast enhanced CT within $48 \mathrm{~h}$ before drainage, $\mathrm{n}=88$ no microbiology, $\mathrm{n}=56$ with no sufficient clinical data for the score calculation, $\mathrm{n}=18$ no serological data, $\mathrm{n}=4 \mathrm{CT}$ texture analysis not possible due to artifacts.

Finally, 320 patients $(\mathrm{n}=119$ females, $35.6 \%$, mean age of $62 \pm 14$ years, range 20-94 years) were included into the analysis. Figure 1 gives an overview of the patient acquisition.

\section{Image analysis}

At first, the $\mathrm{CT}$ images were qualitatively evaluated by one reader using the picture archiving and communication system (Syngo Plaza, Siemens). In unclear cases, a second reader with 5 years of experience in general radiology was consulted, and a consensus was met.

The quantitative evaluation of the CT images was performed accordingly to Gnannt et al. [6].

- The maximum length was measured.

- Hounsfield units (HU) measured with a region of interest (ROI) within the fluid collection

- Presence or absence of a contrast enhancing rim of the fluid collection.

- Presence or absence of fat stranding surrounding the fluid collection.

- Presence or absence of entrapped gas within the collection.

\section{Clinical data acquisition}

The electronic medical records system was utilized to extract the following clinical data: age, sex, underlying disease, presence of medication with immunosuppressive drugs (e.g. glucocorticoids or cytostatics), medication with antibiotics, body temperature and blood sample including CRP, white blood cell counts, procalcitonin and interleukin 6 within $6 \mathrm{~h}$ before percutaneous drainage.

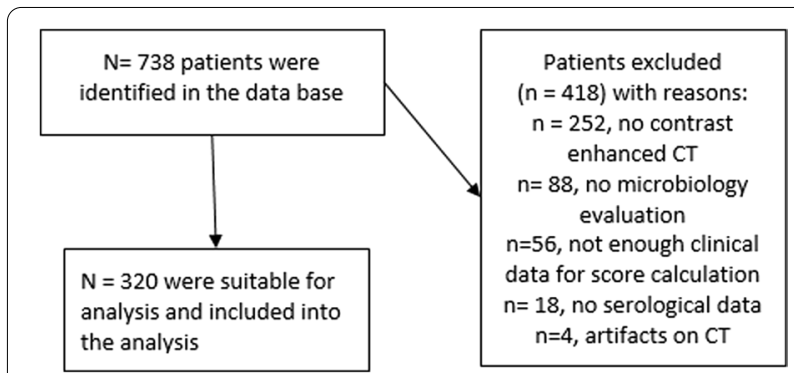

Fig. 1 Flow chart of the acquisition of the patient sample. After exclusion for the given reasons, the final patient sample 320 patients with overall 402 fluid collections were included into the present study 


\section{Image acquisition}

CT imaging was performed in a clinical setting with a 128 or 256 slices CT scanner (Ingenuity or iCT, Philips, Amsterdam, Netherlands). In all cases, $90 \mathrm{~mL}$ of iodinated intravenous contrast medium was given at a rate of 1.5 to $3.5 \mathrm{~mL} / \mathrm{s}$ by a power injector (Medtron $\mathrm{GmbH}$, Germany), with a scan delay of $70 \mathrm{~s}$ after onset of injection for clinical evaluation of septic foci. Typical imaging parameters were $120 \mathrm{kVp}, 150$ to $300 \mathrm{mAs}$, and a slice thickness of $1 \mathrm{~mm}$.

The CT intervention was performed with a 16 slices scanner (Big Bore 16, Philips, Amsterdam, Netherlands). In all patients, a native $\mathrm{CT}$ spiral scan was performed for planning of the intervention covering the body area of the known fluid collection. Typical imaging parameters were $120 \mathrm{kVp}, 150 \mathrm{mAs}$, and a slice thickness of $2 \mathrm{~mm}$.

In all cases, a percutaneous drainage was placed within the fluid collection, size ranging from 8-F to 22-F depending on clinical presenting and localization.

\section{Score calculation}

The previously proposed score by Gnannt et al. was calculated according to the publication [6]. In short, 10 possible points can be obtained. Clinically parameters are known or absent Diabetes, CRP over or under $100 \mathrm{mg} / \mathrm{L}$, gas entrapment, CT attenuation under $10 \mathrm{HU}$, between 10 and $20 \mathrm{HU}$, over $20 \mathrm{HU}$. A score of $0-2$ points was defined for a low probability of infection, 3-10 for a high probability of infection.

The second score by Radosa et al. was also calculated [4]. This score uses only CRP as a serological parameter 0 or 4 points with a cut-off value $(150 \mathrm{mg} / \mathrm{L})$, CT attenuation under or over $20 \mathrm{HU} 0$ or 2 points, gas entrapment 0 or 3 points, wall enhancement 0 or 2 points. The proposed cut-off value is 5 , under for low probability of infection, over for high probability of infection.

\section{Texture analysis}

One the next step, the texture analysis was performed blinded to the microbiological results. CT images further processed with the free available texture analysis software MaZda (version 4.7, available at http://www.eletel.p. lodz.pl/mazda/) [10, 11]. Images were analyzed in $1 \mathrm{~mm}$ soft tissue kernel reconstructed slices. A polygonal ROI was placed on the largest, representative slide of the fluid collection. The ROI was clearly drawn within the boundary of the fluid collection. Moreover, entrapped gas was avoided in the ROI, which could influence the texture analysis results. For each ROI, gray-level normalization was performed, using the limitation of dynamics to $\mu \pm 3$ SD ( $\mu$ gray level mean, SD standard deviation) to minimize the influence of contrast and brightness variation, as it was performed previously $[12,13]$. The extracted texture features are as follows: gray-level histogram (mean, variance, skewness, kurtosis, percentiles $(1,10,50,90$, 99\%), co-occurrence matrix (angular second moment, contrast, correlation, entropy, sum entropy, sum of squares, sum average, sum variance, inverse difference moment, difference entropy, difference variance (for four directions and five interpixel distances (offsets; $n=1$ to 5)), run-length matrix (run-length non-uniformity, gray-level non-uniformity, long run emphasis, short run emphasis, fraction of image in runs)), absolute gradient (gradient mean, variance, skewness, kurtosis, non-zeros), autoregressive model (theta 1 to 4 , sigma), and wavelet transform (energies of wavelet transform coefficients in sub-bands LL, LH, HL, HH). Altogether, 279 texture features were retrieved from every fluid collection.

Figure 2 displays 2 representative cases of the patient sample, one patient with an infected fluid collection and one with a sterile fluid collection.

\section{Microbiological analysis}

All fluid collections were microbiologically analyzed during clinical work up blinded to the imaging results. As part of the CT-guided intervention, at least $5 \mathrm{ml}$ of drained fluid was sent for microbiological analysis. According to current recommendations, fluid collections were considered infected if leukocytes and bacteria were detected on the Gram stain and/or the culture was positive for bacteria or fungi [6]. If these were negative, the fluid collection was classified as non-infected.

\section{Statistical analysis}

Statistical analysis was performed using GraphPad Prism 5 (GraphPad Software, La Jolla, CA, USA) and SPSS STATISTICS (IBM, Version 25.0; Armonk, NY, USA). Collected data were evaluated by means of descriptive statistics (absolute and relative frequencies). Spearman's correlation coefficient ( $r$ ) was used to analyze associations between texture features and the proposed scores. Differences of the texture features between infected and non-infected fluid collections were investigated by two tailed Mann-Whitney test. Then, a Receiver operating characteristics (ROC) analysis was performed and compared between texture features and the scores. AUC characteristics between the scores were compared with DeLong test. A Random-Forest classifier as well as a multivariate analysis was used to construct a predictive model to classify infected fluid collections using qualitative as well as quantitative imaging parameters and serological parameters. In all instances, $p$ values $<0.05$ were taken to indicate statistical significance. 

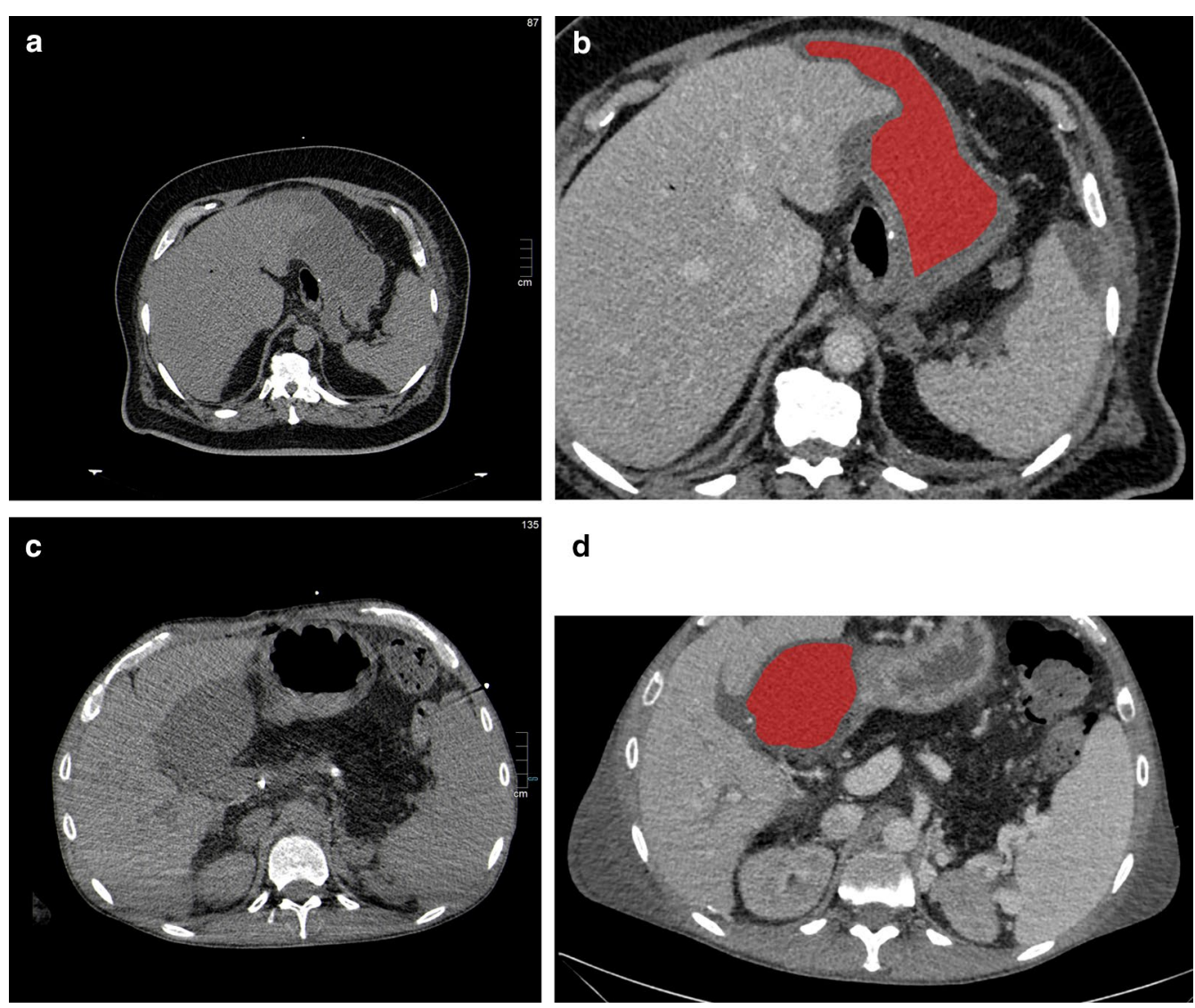

d

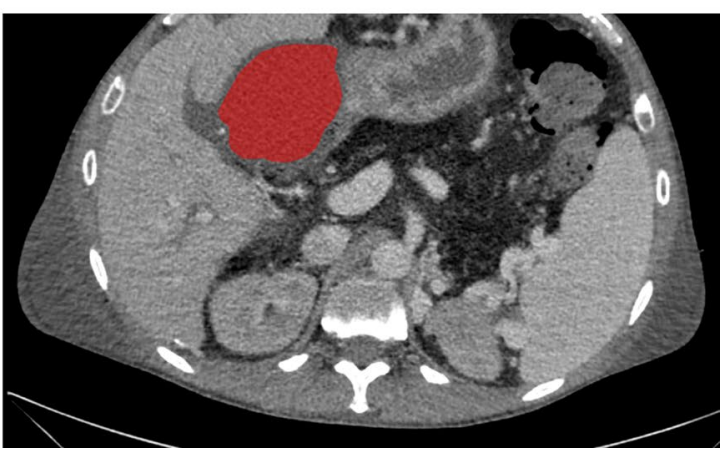

Fig. 2 a Representative 66-years old male case of the patient sample with an infected perigastric fluid collection after sleeve-gastrectomy surgery 5 days ago. A rim contrast media enhancement and higher Hounsfield units above 20 can be appreciated. There are no gas entrapments or perifocal stranding. Small free perisplenic free fluid can also be seen. $\mathbf{b}$ The drawn region of interest of the fluid collection. $\mathbf{c}$ Representative 54-years old male case with a non-infected fluid collection. The fluid collection is located at the hilum of the liver after liver transplantation 4 days ago. No contrast media enhancement, gas entrapment, perifocal stranding or higher Hounsfield units can be appreciated. $\mathbf{d}$ The drawn region of interest of the fluid collection

\section{Results}

\section{Clinical and radiological parameters}

An overview of the descriptive statistics of the investigated demographic, serologic and imaging parameters is given by Table 1 .

Overall, 402 fluid collections were investigated. In most patients one fluid collection was identified, in 68 cases 2 fluid collections were identified and in 7 cases 3 fluid collections were identified. There were 147 non-infected (36.7\%) and 255 infected fluid collections (63.3\%). In all patients, a successful percutaneous CT-guided drainage was placed within the fluid collection and the fluid was microbiologically evaluated.

Table 2 gives an overview of the underlying diseases of the patients and localizations of the fluid collections. In $52.2 \%$ of all patients, a malignant tumor was known, $30 \%$ of all patients suffered from a septic disease.

\section{Diagnostic accuracy of the investigated scores}

The resulting score was only defined by imaging parameters (Table 3) in the multilinear regression: No serological or clinical parameters were utilized by this analysis.

The accuracy of the investigated scores is shown by Table 4 . The present score achieved a sensitivity of 0.78 , a specificity of 0.69 with an AUC 0.82 .

Gnannt et al. achieved an AUC of 0.81 (95\% CI $0.77-$ 0.85 ), Radosa et al. an AUC of 0.75 (95\% CI 0.70-0.79) and the present score an AUC of 0.82 (95\% CI 0.78-0.86) (Fig. 3a). The present score and the score by Gnannt et al. were both significantly better than the score by Radosa et al.

The present score was significantly superior to the score by Radosa et al. $(p=0.04)$, and not inferior to the score by Gnannt et al. $(p=0.23)$. The score by Gnannt et al. was significantly better than the score by Radosa 
Table 1 Overview of the demographic, serologic and imaging parameters of the patient sample

\begin{tabular}{|c|c|c|c|}
\hline Parameter & $\begin{array}{l}\text { Non-infected fluid collections } \\
(n=147)\end{array}$ & Infected fluid collections $(\mathrm{n}=255$ ) & $p$ value \\
\hline Age, years & $62 \pm 15(21-94)$ & $62 \pm 14(20-90)$ & 0.9 \\
\hline Sex, male/female & $70 / 37$ & $136 / 77$ & 0.4 \\
\hline Diabetes & $26(21.5 \%)$ & $63(29.6 \%)$ & 0.07 \\
\hline Immunosuppressive drugs & $16(13.2 \%)$ & $31(14.6 \%)$ & 0.4 \\
\hline Antibiotics & $92(76 \%)$ & $172(80.8 \%)$ & 0.2 \\
\hline C-reactive protein, $\mathrm{mg} / \mathrm{L}$ & $152 \pm 101(1-444)$ & $177 \pm 102(1-435)$ & 0.02 \\
\hline Leukocytes, $10^{9} / \mathrm{L}$ & $13.4 \pm 6.9(0.7-38.5)$ & $15.3 \pm 8(2.3-81.2)$ & 0.009 \\
\hline Procalcitonin, ng/ml & $3.2 \pm 8.1(0.1-41.4)$ & $6 \pm 15.6(0.1-108.5)$ & 0.02 \\
\hline Interleukin-6, pg/ml & $431 \pm 701(38-1850)$ & $3061 \pm 6953(38-28,687)$ & 0.4 \\
\hline Attenuation, $\mathrm{HU}$ & $13 \pm 11(0-52)$ & $18 \pm 8(0-58)$ & $<0.001$ \\
\hline Maximum diameter, $\mathrm{cm}$ & $8.5 \pm 3.9(2.2-25.5)$ & $7.8 \pm 3.4(2.1-20)$ & 0.07 \\
\hline Wall enhancement & $59(40.1 \%)$ & $153(60 \%)$ & $<0.001$ \\
\hline Fat stranding & $57(38.8 \%)$ & $161(63.1 \%)$ & $<0.001$ \\
\hline Entrapped gas & $30(20.4 \%)$ & $148(58 \%)$ & $<0.001$ \\
\hline
\end{tabular}

Data are expressed as mean \pm standard deviation (range) or frequencies with percentages $p$ values are calculated with Mann-Whitney Test or Fisher's exact test where appropriate $\mathrm{HU}=$ Hounsfield Units

Table 2 Overview of the underlying diseases and localization of the fluid collection

\begin{tabular}{ll}
\hline Underlying disease & Patients \\
\hline Malignancy & $167(52.2 \%)$ \\
Infection & $96(30 \%)$ \\
Trauma & $8(2.5 \%)$ \\
Vascular & $12(3.8 \%)$ \\
Others & $37(11.5 \%)$ \\
Localization & \\
Intra- and extraperitoneal cavity & $112(35 \%)$ \\
Pleural & $89(27.8 \%)$ \\
Liver & $63(19.7 \%)$ \\
Pelvic & $39(12.2 \%)$ \\
Others & $17(5.3 \%)$ \\
\hline
\end{tabular}

et al. $(p=0.002)$. Figure 3 a shows the corresponding ROC graphs.

There was a strong positive correlation between the score by Gnannt et al. with the score by Radosa et al. $(\mathrm{r}=0.63, p<0.001)$. The present score correlated both with Gnannt et al. $(\mathrm{r}=0.80, p<0.001)$ and the score by Radosa et al. ( $r=0.70, p<0.001)$.

\section{Diagnostic accuracy for postoperative and pleural fluid collection}

Subanalyses were performed to further assess the diagnostic accuracy of the scores.
Table 3 The new proposed score identified by multivariate regression analysis

\begin{tabular}{lll}
\hline Present score & $\boldsymbol{\beta}$ (regression coefficient) & Points \\
\hline Fat stranding & & \\
No & 1.6 & 0 \\
Yes & & 2 \\
Gas entrapment & 0 & 0 \\
No & 3 & 3 \\
Yes & & \\
CT attenuation, HU & 0 & 0 \\
$\leq 10$ & 4 & 4 \\
$>10$ & & 0 \\
Minimum total score & & 9 \\
Maximum total score & & \\
Cut-off $\geq 5$ points & &
\end{tabular}

$\mathrm{HU}=$ Hounsfield units

Table 4 Comparison between the analyzed scores in regard of diagnostic accuracy

\begin{tabular}{lll}
\hline & AUC & $\mathbf{9 5 \% ~ C l}$ \\
\hline Gnannt score & 0.81 & 0.77 to 0.85 \\
Radosa score & 0.75 & 0.70 to 0.79 \\
Present score & 0.82 & 0.78 to 0.86 \\
\hline
\end{tabular}

$\mathrm{AUC}=$ area under curve, $\mathrm{Cl}=$ Confidence interval 

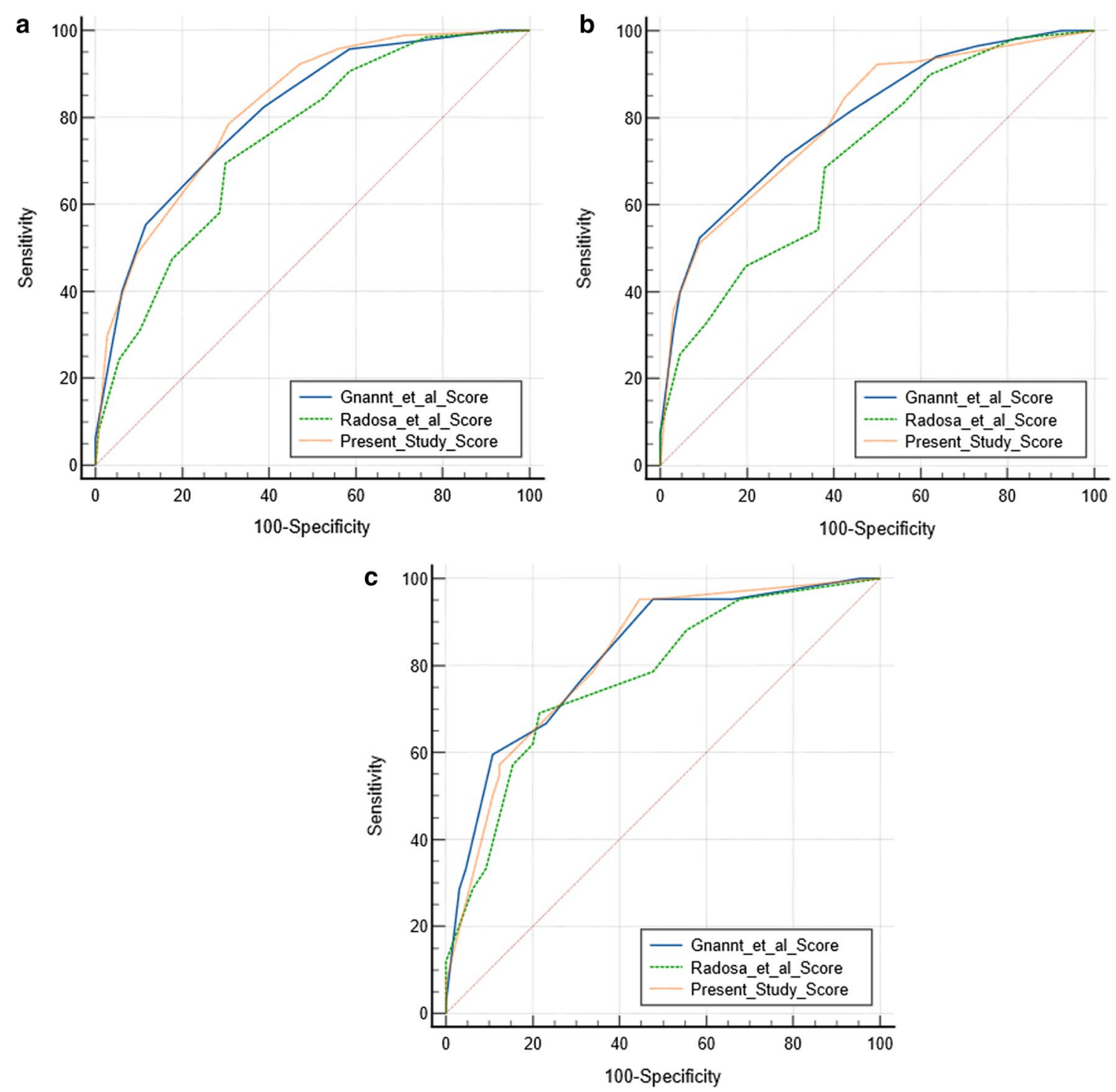

Fig. 3 a ROC curves of the investigated CT scores in the overall patient sample. Gnannt et al. achieved an AUC of 0.81, Radosa et al. an AUC of 0.75 and the present score an AUC of 0.82. The present score and the score by Gnannt et al. were both significantly better than the score by Radosa et al. b ROC curve of the investigated CT score in the subanalysis of only postoperative patients. Gnannt et al. achieved an AUC of 0.80, Radosa et al. an AUC of 0.71 and the present score an AUC of 0.80. The present score and the score by Gnannt et al. were both significantly better than the score by Radosa et al. c ROC curve of the investigated CT score in the subanalysis of pleural fluid collections. Gnannt et al. achieved an AUC of 0.82, Radosa et al. an AUC of 0.77 and the present score an AUC of 0.82

Overall, 234 fluid collections were included into the analysis only investigating postoperative fluid collections.

The AUC values did not change significantly: Gnannt et al. achieved an AUC of 0.80 (95\% CI 0.74-0.85), Radosa et al. an AUC of 0.71 (95\% CI 0.65-0.77) and the present score an AUC of 0.80 (95\% CI 0.74-0.85) (Fig. 3b). The present score and the score by Gnannt et al. were both significantly better than the score by Radosa et al.

Overall, 107 fluid collections were included into the analysis of pleural fluid collections.

Again, the AUC values did not change significantly: Gnannt et al. achieved an AUC of 0.82 (95\% CI 0.740.89), Radosa et al. an AUC of 0.77 (95\% CI 0.68-0.85) 
and the present score an AUC of 0.82 (95\% CI 0.73-0.89) (Fig. 3c).

\section{Accuracy of imaging and clinical findings}

Table 5 gives an overview of the accuracy to predict infected fluid collections of single imaging and clinical features. CRP was the best clinical feature achieving a sensitivity of 0.76 and a specificity of 0.40 employing a threshold value of $114 \mathrm{mg} / \mathrm{dl}$. The best imaging feature was HU, which achieved a sensitivity of 0.87 and a specificity of 0.55 employing a threshold value of $11 \mathrm{HU}$.

\section{Texture analysis parameters}

Several texture features were significantly different between infected and non-infected fluid collections. Table 6 gives an overview of the statistically significant features. However, no texture features achieved a better AUC than the conventional imaging features. Therefore, no texture feature was included into the proposed score.

\section{Discussion}

The present study elucidated whether CT scores can reliably predict infection of a fluid collection undergoing percutaneous CT drainage. A novel score was provided only utilizing CT findings, which was comparably good in regard of accuracy to the published scores. Another finding was that texture analysis derived from $\mathrm{CT}$ images does not provide additional value to diagnose infected fluid collections.

Previously, several CT findings were identified to be indicative of infected fluid collection comprising increased attenuation, encapsulation with or without wall enhancement, presence of gas entrapment, and stranding of the surrounding tissue $[3,4,6]$. However, it is also widely acknowledged that these findings considered separately cannot reliably predict the infection of a fluid collection. This was previously shown in a study on 92

Table 5 Comparison of the diagnostic accuracy of the clinical and imaging features

\begin{tabular}{lllll}
\hline Parameter & $\begin{array}{l}\text { Threshold- } \\
\text { value }\end{array}$ & Sensitivity & Specificity & Youden-Index \\
\hline BMI & $28.2 \mathrm{~kg} / \mathrm{mm}^{2}$ & 0.30 & 0.80 & 0.09 \\
Leucocytes & $14.810^{9} / \mathrm{L}$ & 0.49 & 0.67 & 0.16 \\
CRP & $114 \mathrm{mg} / \mathrm{L}$ & 0.76 & 0.40 & 0.16 \\
Fat stranding & Positive & 0.63 & 0.61 & 0.24 \\
Air entrap- & Positive & 0.58 & 0.80 & 0.38 \\
ment & & 0.60 & 0.60 & 0.20 \\
Enhance- & Positive & 0.60 & \\
ment & & 0.87 & 0.55 & 0.42 \\
HU & 10.5 & & & \\
\hline
\end{tabular}

$\mathrm{HU}=$ Hounsfield Units, $\mathrm{BMI}=$ Body mass index, $\mathrm{CRP}=\mathrm{C}$-reactive protein
Table 6 Overview of the statistically significant texture parameters between infected and non-infected fluid collections derived from the contrast enhanced $\mathrm{CT}$

\begin{tabular}{|c|c|}
\hline Texture feature & $p$ value \\
\hline Mean & $<0.001$ \\
\hline Kurtosis & 0.016 \\
\hline Perc,01\% & $<0.001$ \\
\hline Perc, $10 \%$ & $<0.001$ \\
\hline Perc,50\% & $<0.001$ \\
\hline Perc, $90 \%$ & $<0.001$ \\
\hline Perc,99\% & 0.017 \\
\hline _Area_S(1,0) & $<0.001$ \\
\hline _Area_S(0,1) & $<0.001$ \\
\hline _Area_S(1,1) & $<0.001$ \\
\hline _Area_S(1,-1) & $<0.001$ \\
\hline $\begin{array}{l}S(1,-1) \\
\text { InvDfMom }\end{array}$ & 0.011 \\
\hline _Area_S(2,0) & $<0.001$ \\
\hline $\begin{array}{l}\mathrm{S}(2,0) \\
\text { InvDfMom }\end{array}$ & 0.045 \\
\hline _Area_S(0,2) & $<0.001$ \\
\hline _Area_S(2,2) & $<0.001$ \\
\hline _Area_S(2,-2) & $<0.001$ \\
\hline $\begin{array}{l}S(2,-2) \\
\text { InvDfMom }\end{array}$ & 0.011 \\
\hline _Area_S(3,0) & $<0.001$ \\
\hline _Area_S(0,3) & $<0.001$ \\
\hline _Area_S(3,3) & $<0.001$ \\
\hline _Area_S(3,-3) & $<0.001$ \\
\hline $\begin{array}{l}S(3,-3) \\
\text { InvDfMom }\end{array}$ & 0.026 \\
\hline _Area_S(4,0) & $<0.001$ \\
\hline $\begin{array}{l}S(4,0) \\
\text { InvDfMom }\end{array}$ & 0.045 \\
\hline _Area_S(0,4) & $<0.001$ \\
\hline S(0,4)Entropy & 0.046 \\
\hline _Area_S(4,4) & $<0.001$ \\
\hline _Area_S(4,-4) & $<0.001$ \\
\hline _Area_S(5,0) & $<0.001$ \\
\hline _Area_S(0,5) & $<0.001$ \\
\hline$S(0,5)$ Entropy & 0.046 \\
\hline _Area_S(5,5) & $<0.001$ \\
\hline S(5,5)Entropy & 0.045 \\
\hline _Area_S(5,-5) & $<0.001$ \\
\hline S(5,-5)Entropy & 0.037 \\
\hline $\begin{array}{l}\text { Horzl_RLNonU } \\
\text { ni }\end{array}$ & $<0.001$ \\
\hline $\begin{array}{l}\text { Horzl_GLevNon } \\
\text { U }\end{array}$ & $<0.001$ \\
\hline $\begin{array}{l}\text { Horzl_LngREm } \\
\text { ph }\end{array}$ & 0.006 \\
\hline $\begin{array}{l}\text { Horzl_ShrtREm } \\
\text { p }\end{array}$ & 0.034 \\
\hline Horzl_Fraction & 0.015 \\
\hline
\end{tabular}


Table 6 (continued)

\begin{tabular}{lr}
\hline Texture feature & $\boldsymbol{p}$ value \\
\hline Vertl_RLNonUn & $<0.001$ \\
i & \\
Vertl_GLevNon & $<0.001$ \\
U & \\
Vertl_LngREmp & 0.040 \\
h & \\
45dgr_RLNonU & $<0.001$ \\
ni & \\
45dgr_GLevNo & $<0.001$ \\
nU & \\
45dgr_LngREm & 0.044 \\
ph & \\
135dr_RLNonU & $<0.001$ \\
ni & \\
135dr_GLevNo & $<0.001$ \\
nU & \\
135dr_LngREm & 0.003 \\
ph & \\
135dr_ShrtRE & 0.017 \\
mp & \\
135dr_Fraction & 0.008 \\
_AreaGr & $<0.001$ \\
GrSkewness & 0.030 \\
GrKurtosis & 0.003 \\
GrNonZeros & 0.030 \\
_AreaARM & 0.001 \\
Sigma & 0.048 \\
WavEnHH_s-2 & 0.022 \\
WavEnHL_s-4 & 0.011 \\
WavEnHH_s-5 & \\
WavEnHH_s-7 & \\
\hline & \\
&
\end{tabular}

patients with postoperative fluid collections achieving an average sensitivity of $83.4 \%$ and a specificity of $39.3 \%$ utilizing the features gas entrapment and high attenuation fluid (20 or greater HU) [3].

That is why the previously proposed $2 \mathrm{CT}$ scores are based upon a set of imaging features to achieve a better accuracy to diagnose infected fluid collections [4, 6]. Moreover, with combination of imaging and clinical parameters the diagnostic accuracy can be improved.

One rationale of the present study was that texture analysis could better characterize fluid collections due to its ability to quantify heterogeneities of radiological images [7].

This has been extensively shown for oncologic imaging with papers elucidating the possible use of texture analysis to reflect histopathology and microstructure of tumors [7-9]. However, the present analysis can state that texture analysis of CT images is not superior to discriminate infected of non-infected fluid collections compared to conventional imaging findings.
It is widely acknowledged that higher attenuation of a fluid collection is mainly caused by debris [2, 6]. Hemorrhage is another reason, which can result in a higher attenuation in non-infected fluid collections. Yet, higher attenuation over 10 Hounsfield units is the strongest predictor of infected fluid collections, with a good sensitivity and specificity, which is almost as good as the combined scores.

Presumably, debris and other associated findings of an abscess or infected fluid collection do not cause distinctive alterations of texture features in comparison to sterile hematomas and seromas, which can explain the negative results.

Another part of the study was the validation of the two proposed CT scores by Gnannt et al. and Radosa et al. [4, 6]. These scores utilized the conventional imaging findings of an infected fluid collection and CRP as a serological parameter. Gnannt et al. also used the anamnestic feature of a known diabetes, which was not needed by Radosa et al.

Contrary to the previous studies, the present study included pleural fluid collections suspicious for empyema and fluid collections in non-surgical patients into the analysis. This results in a larger patient spectrum but with the same important clinical question, whether a fluid collection is infected or not. Our results can therefore be considered as representative for all fluid collections, which are considered for CT-guided percutaneous drainage. To adjust for better comparability, the subanalyses were performed for postoperative abdominal fluid collections and pleural fluid collections separately. Nonetheless, both analyses showed similar results.

The new proposed score only needs imaging criteria and was more accurate as the score by Radosa et al. and not statistically inferior as the more complex score by Gnannt et al. The new score could be used in clinical routine to guide treatment indication without any need of further anamnestic and/or serological information.

CRP was discussed as the most important clinical parameter to diagnose infected fluid collection [4, 14]. In a clinical case series on surgical patients, the combination of an increased CRP level together with the CT could discriminate patients with a major complication to those without [14]. The proposed threshold was $200 \mathrm{mg} / \mathrm{L}$ in this mentioned study is higher compared to the used one of $150 \mathrm{mg} / \mathrm{L}$ in the study by Radosa et al. and the threshold of $100 \mathrm{mg} / \mathrm{L}$ used by Gnannt et al. The present analysis identified the best accuracy with a threshold of $114 \mathrm{mg} / \mathrm{L}$.

It must be acknowledged that a superior CT based diagnosis of infected fluid collections can reduce possible drainage overtreatment. However, there are also noninfected fluid collections, which might need drainage 
treatment. For example, non-infected fluid collections with possible mass effect causing symptoms. Yet even for those cases, it could be useful for treatment planning to know the infection state of the fluid collection. For example, choice of drainage type could be influenced by this.

There are several limitations of the present study to address. First, it is a retrospective study with possible known inherent bias. However, imaging and microbiology analyses were performed independently. Second, some serological inflammation parameters, including procalcitonin and Interleukin-6 were not available for all patients, which reflects clinical routine. Moreover, for every patient leucocyte count and CRP were available for analysis, which are most commonly used. Third, possible selection bias can be assumed as we only could include fluid collections undergoing CT-guided drainage treatment. There might be fluid collections not treated by this procedure due to low likelihood for infection or small size without possible drainage placement. Fourth, the imaging was analyzed by one reader. However, it was shown that an excellent interreader agreement can be assumed for the typical findings of infected fluid collections, which reduces possible bias [6]. Fifth, one should consider that preprocessing is important for texture analysis results $[15,16]$. There was no complex preprocessing in the present analysis, which could have an influence on the results.

\section{Conclusions}

Albeit texture features derived from CT images were associated with the presence of infection of fluid collections, these were not superior to conventional imaging findings. A score was provided based upon imaging parameters alone, which was comparable to the published scores that are dependent on clinical and serological information. These findings could reduce the need for drainage therapy, which needs to be prospectively evaluated.

\section{Abbreviations \\ CT: Computed tomography; ROI: Region of interest; HU: Hounsfield Unit; CRP: C-reactive protein.}

\section{Acknowledgements}

None.

\section{Authors' contributions}

H-JM, BS: study concept and design, data acquisition, data analysis and inter- pretation, and writing of manuscript. MM, JL, SE, HG, TD: data analysis, interpretation and revision of manuscript for important intellectual content. All authors read and approved the final manuscript.

\section{Funding}

Open Access funding enabled and organized by Projekt DEAL.

\section{Availability of data and materials}

The datasets used and ana-yzed during the current study are available from the corresponding author on reasonable request.

\section{Declarations}

Ethics approval and consent to participate

Following the Declaration of Helsinki, the study was approved as a retrospective human study by the Institutional Review Board (University of Leipzig). The informed consent of patient, parents, or guardians was waived. All methods were carried out in accordance with relevant guidelines and regulations.

\section{Consent for publication}

Not applicable.

\section{Competing interests}

The authors have no competing interest to declare.

\section{Author details}

${ }^{1}$ Department of Diagnostic and Interventional Radiology, University of Leipzig, Liebigstraße 20, 04103 Leipzig, Germany. ${ }^{2}$ Department of Visceral, Transplant,

Thoracic and Vascular Surgery, University of Leipzig, Leipzig, Germany.

Received: 13 October 2021 Accepted: 17 November 2021

Published online: 06 December 2021

\section{References}

1. Brook I. Microbiology and management of abdominal infections. Dig Dis Sci. 2008;53(10):2585-91. https://doi.org/10.1007/s10620-007-0194-6 (Epub 2008 Feb 21 PMID: 18288616).

2. Sirinek KR. Diagnosis and treatment of intra-abdominal abscesses. Surg Infect (Larchmt). 2000;1 (1):31-8. https://doi.org/10.1089/1096296003 21272 (PMID: 12594907)

3. Allen BC, Barnhart H, Bashir M, Nieman C, Breault S, Jaffe TA. Diagnostic accuracy of intra-abdominal fluid collection characterization in the era of multidetector computed tomography. Am Surg. 2012;78(2):185-9 (PMID: 22369827)

4. Radosa CG, Radosa JC, Laniado M, Brandt J, Streitzig J, Seppelt D, et al. Infected versus sterile abdominal fluid collections in postoperative CT: a scoring system based on clinical and imaging findings. Abdom Radiol (NY). 2020;45(9):2871-8. https://doi.org/10.1007/s00261-020-02635-y (PMID: 32671442)

5. Men S, Akhan O, Köroğlu M. Percutaneous drainage of abdominal abcess. Eur J Radiol. 2002;43(3):204-18. https://doi.org/10.1016/s0720-048x(02) 00156-0 (PMID: 12204403)

6. Gnannt R, Fischer MA, Baechler T, Clavien PA, Karlo C, Seifert B, et al. Distinguishing infected from noninfected abdominal fluid collections after surgery: an imaging, clinical, and laboratory-based scoring system. Invest Radiol. 2015;50(1):17-23. https://doi.org/10.1097/RLI.0000000000000090 (PMID: 25198832)

7. Incoronato M, Aiello M, Infante T, Cavaliere C, Grimaldi AM, Mirabelli P, et al. Radiogenomic analysis of oncological data: a technical survey. Int J Mol Sci. 2017;18(4):805. https://doi.org/10.3390/ijms18040805.PMID: 28417933;PMCID:PMC5412389.

8. Wu G, Jochems A, Refaee T, Ibrahim A, Yan C, Sanduleanu S, Woodruff HC, Lambin P. Structural and functional radiomics for lung cancer. Eur J Nucl Med Mol Imaging. 2021. https://doi.org/10.1007/s00259-021-05242-1 (Epub ahead of print. PMID: 33693966).

9. Just N. Improving tumour heterogeneity MRI assessment with histograms. Br J Cancer. 2014;111(12):2205-13. https://doi.org/10.1038/bjc. 2014.512 (PMID: 25268373).

10. Strzelecki M, Szczypinski P, Materka A, Klepaczko A. A software tool for automatic classification and segmentation of 2D/3D medical images. Nucl Instrum Methods Phys Res A. 2013;702:137-40.

11. Szczypiński PM, Strzelecki M, Materka A, Klepaczko A. MaZda—a software package for image texture analysis. Comput Methods Prog Biomed. 2009;94:66-76. 
12. Fruehwald-Pallamar J, Czerny C, Holzer-Fruehwald L, Nemec SF, MuellerMang C, Weber M, Mayerhoefer ME. Texture-based and diffusionweighted discrimination of parotid gland lesions on MR images at 3.0 Tesla. NMR Biomed. 2013;26(11):1372-9. https://doi.org/10.1002/nbm. 2962 (PMID: 23703801).

13. Meyer HJ, Renatus K, Höhn AK, Hamerla G, Schopow N, Fakler J, Josten C, Surov A. Texture analysis parameters derived from T1-and T2-weighted magnetic resonance images can reflect Ki67 index in soft tissue sarcoma. Surg Oncol. 2019;30(9):92-7. https://doi.org/10.1016/j.suronc.2019.06. 006.

14. Straatman J, Cuesta MA, Gisbertz SS, Van der Peet DL. Value of a step-up diagnosis plan: CRP and CT-scan to diagnose and manage postoperative complications after major abdominal surgery. Rev Esp Enferm Dig. 2014;106(8):515-21 (PMID: 25544408).

15. Versaci M, Calcagno S, Morabito FC. Fuzzy geometrical approach based on unit hyper-cubes for image contrast enhancement. In: 2015 IEEE international conference on signal and image processing applications (ICSIPA). 2015; 488-493. doi: https://doi.org/10.1109/ICSIPA.2015.7412240

16. Larue RT, Defraene G, De Ruysscher D, Lambin P, van Elmpt W. Quantitative radiomics studies for tissue characterization: a review of technology and methodological procedures. Br J Radiol. 2017;90(1070):20160665. https://doi.org/10.1259/bjr.20160665.

\section{Publisher's Note}

Springer Nature remains neutral with regard to jurisdictional claims in published maps and institutional affiliations.

- fast, convenient online submission

- thorough peer review by experienced researchers in your field

- rapid publication on acceptance

- support for research data, including large and complex data types

- gold Open Access which fosters wider collaboration and increased citations

- maximum visibility for your research: over $100 \mathrm{M}$ website views per year

At BMC, research is always in progress.

Learn more biomedcentral.com/submissions 